\title{
chaJOPEN
}

\section{Extent of a valsartan drug shortage and its effect on antihypertensive drug use in the Canadian population: a national cross-sectional study}

\author{
Jennifer Fenna PharmD MHS, Cherry Chu MSc, Rola Hassan PharmD, Tara Gomes MHSc PhD, \\ Mina Tadrous PharmD PhD
}

\section{Abstract}

Background: Drug shortages represent a growing global problem, with potentially serious consequences to patients and the health care system. Our study investigates the impacts of a major recall and shortage of valsartan, an angiotensin receptor blocker (ARB), in July 2018 in Canada.

Methods: We conducted a time-series analysis of antihypertensive drugs dispensed in Canada between 2015 and 2019 using commercially available retail prescription data. Using autoregressive integrated moving average (ARIMA) modelling, we evaluated the change in valsartan use after the recall. We also measured the overall use of ARBs, angiotensin-converting-enzyme (ACE) inhibitors and other antihypertensive drug classes for the same period.

Results: After the recall in July 2018, valsartan use decreased $57.8 \%$, from 362231 prescriptions dispensed in June 2018 to 152892 in September 2018 (difference $=209339, p<0.0001$ ). Overall use of the ARB drug class decreased $2.0 \%$, from 1577509 prescriptions dispensed in June 2018 to 1545591 in September 2018 (difference $=31918, p=0.0003$ ), but use of non-valsartan ARBs increased 14.6\%, from 1215278 to 1392699 prescriptions dispensed (difference $=177421, p<0.0001$ ) in the same time frame. Although use of ACE inhibitors initially declined, this reduction was not sustained. The valsartan recall was not associated with a significant impact on use of other antihypertensive drug classes.

Interpretation: Our findings illustrate the impact of a major drug shortage, with the immediate and substantial reduction of valsartan dispensed and cascading effects on other ARBs, though future research is warranted to understand the consequences of such extensive shortages on clinical outcomes and health system costs. Improved policy strategies are needed to address the underlying causes of drug shortages and to mitigate their effects.

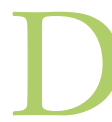

rug shortages are a concerning global issue, ${ }^{1,2}$ with annual shortages of over 1000 in Canada,,$^{1,3}$ and an increasing impact on drugs used to treat cardiovascular disease. ${ }^{2,4}$ Disruptions to drug availability and the accompanying increase in demand of alternative therapies has potentially serious implications for patients, clinicians and the health care system., 3

Finding suitable alternatives for drugs in short supply increases the workload of clinicians and pharmacists, and changes in therapy introduce additional safety risks related to medication errors or adverse events. ${ }^{2,6}$ Patients may experience impaired health, stress and anxiety ${ }^{6}$ as well as suboptimal therapy with less viable alternatives ${ }^{2}$ or disruption of therapy; ${ }^{7}$ there may also be risk of therapeutic failure with medication switches, leading to increased medical visits and hospitalizations. ${ }^{6-8}$ The economic implications on the health care system include labour costs to manage shortages and, potentially, more expensive costs of alternative drugs. ${ }^{2}$ Despite being a well-recognized global issue, the extent of the impact and clinical consequences of drug shortages on patient care remains relatively unknown. ${ }^{6,9}$
Causes of drug shortages can be complex and multifactorial, ${ }^{3}$ with an example being the recall of valsartan, an angiotensin receptor blocker (ARB) drug that is predominantly used to treat hypertension and heart failure. ${ }^{10}$ In July 2018, Health Canada announced a broad recall of 6 generic valsartan products because of a carcinogenic impurity, $\mathrm{N}$-nitrosodimethylamine (NDMA), thought to have resulted from a change in the manufacturing process of valsartan's active pharmaceutical ingredient by the supplier, Zhejiang Huahai Pharmaceuticals. ${ }^{10}$ In August 2018 and November 2018, the recall was further expanded to include most available

Competing interests: None declared.

This article has been peer reviewed.

Correspondence to: Mina Tadrous, mina.tadrous@utoronto.ca CMAJ Open 2021 December 7. DOI:10.9778/cmajo.20200232 
valsartan products in the Canadian market. ${ }^{11}$ Although viable clinical alternatives exist for valsartan, ${ }^{12}$ multiple other drugs from the ARB class of medications also had shortages in the same year as demand increased as a result of patients switching from valsartan; ${ }^{13}$ this caused a substantial challenge for clinicians seeking to find suitable alternatives in a short time frame. , $14,15^{-15}$

The large magnitude of this drug supply disruption presents an important opportunity to understand the true extent of its impact in terms of the duration of the shortage and the quantity of medications available to patients in Canada. A recent study, limited to publicly funded recipients in Ontario, found that $84.4 \%$ of patients using valsartan moved to an alternative drug in the first month after the recall; ${ }^{7}$ however, little is known about the national extent of the recall. We set out to investigate the effects of the recall and shortage on use of valsartan, as well as other antihypertensive drugs, in Canada.

\section{Methods}

\section{Study design}

We conducted a population-based time-series analysis of antihypertensive drugs dispensed in Canada between January 2015 and December 2019 using the IQVIA Geographic Prescription Monitor data set. We explored the change in use of antihypertensive drugs after the Health Canada recall of valsartan on July 9, 2018.

\section{Data source}

The source of information supporting the IQVIA Geographic Prescription Monitor is the IQVIA retail prescription database, which contains prescription transactions from Canadian drug stores for all dispensed branded and generic products. At the national level, over $75 \%$ of total prescriptions dispensed are captured by a panel of about 6100 retail pharmacies. The monthly estimates are created using IQVIA's patented geospatial projection methodology. These projections are representative of drug use and cost at the provincial and national levels. At the provincial level, the error in estimates of projected use can reach slightly higher levels, although it does not generally exceed $5 \%$ to $10 \%$ (Josiane Gaudet, IQVIA: personal communication, Aug. 5, 2020). The IQVIA database has been previously validated as having high positive predictive value, ${ }^{16,17}$ and is commonly used for research purposes. ${ }^{18,19}$

\section{Statistical analysis}

We determined the use of antihypertensives by calculating the number of prescriptions dispensed nationally on a monthly basis. We stratified all dispensed antihypertensives by individual drug and categorized them into all ARBs, valsartan (defined as both single entity and combination valsartanhydrochlorothiazide, excluding valsartan-sacubitril products), other (non-valsartan) ARBs, angiotensin-converting-enzyme (ACE) inhibitors or other antihypertensive classes (i.e., $\beta$-blockers, calcium channel blockers and diuretics). We also measured the average number of tablets dispensed per prescription of valsartan in the period 3 months before and after the date of the recall to explore the stability of the change in the volume of drugs dispensed because of the recall. As the ARB drugs are taken once daily, this value was used to ascertain the average days of supply dispensed per prescription.

We used interventional autoregressive integrated moving average (ARIMA) models to evaluate the statistical significance of the recall on the prescription volume of each of the antihypertensive drug classes. These models are commonly used to analyze the effect of change (e.g., program intervention) in a time-series analysis. ${ }^{20} \mathrm{We}$ incorporated a step intervention function in the models to test for immediate changes when the valsartan recall occurred in July 2018. We converted all time-series models to stationary series by first-differencing, whereby stationarity was confirmed using the augmented Dickey-Fuller test. ${ }^{21}$

We selected the model parameters using residual autocorrelation function (ACF), partial ACF and inverse ACF correlograms. We assessed model fit using ACF, partial ACF and inverse ACF plots, as well as white noise probability plots, Ljung-Box $\chi^{2}$ tests for white noise and model fit statistics, such as the Akaike information criterion, the Bayesian information criterion and $R^{2}{ }^{20,22} \mathrm{We}$ considered $p$ values less than 0.05 statistically significant, and completed all analyses using SAS Enterprise Guide, version 6.1.

\section{Ethics approval}

The dispensing data used in the study contains no personal health information or patient-specific identifiers, and therefore use of the data did not require review by a research ethics board.

\section{Results}

\section{Angiotensin receptor blockers}

After the recall on July 9, 2018, the volume of dispensed prescriptions for the ARB drug class declined by $2.0 \%$, from 1577509 prescriptions dispensed in June 2018 to 1545591 in September 2018 (difference $=31918$ prescriptions) (Figure 1, Table 1). After this time, there was an increase of $13.5 \%$ to 1753924 prescriptions dispensed in December 2019. The change in ARB prescriptions from the valsartan recall in July 2018 was statistically significant $(p=0.0003)$.

\section{Valsartan}

After the recall, the volume of valsartan prescriptions dispensed declined 57.8\%, from 362231 in June 2018 to 152892 in September 2018 (difference = 209339 prescriptions) (Figure 1, Table 1). Use then increased $13.3 \%$ to 173224 prescriptions in December 2019, for an overall decline of $47.8 \%$ from June 2018. The change in valsartan prescriptions from the recall in July 2018 was statistically significant $(p<0.0001)$. The average supply of valsartan prescriptions dispensed was similar before (April 2018 to June 2018) and after (July 2018 to September 2018) the recall at 40 tablets and 38 tablets per prescription, respectively, showing no major shifts in prescription duration around the time of the recall. 


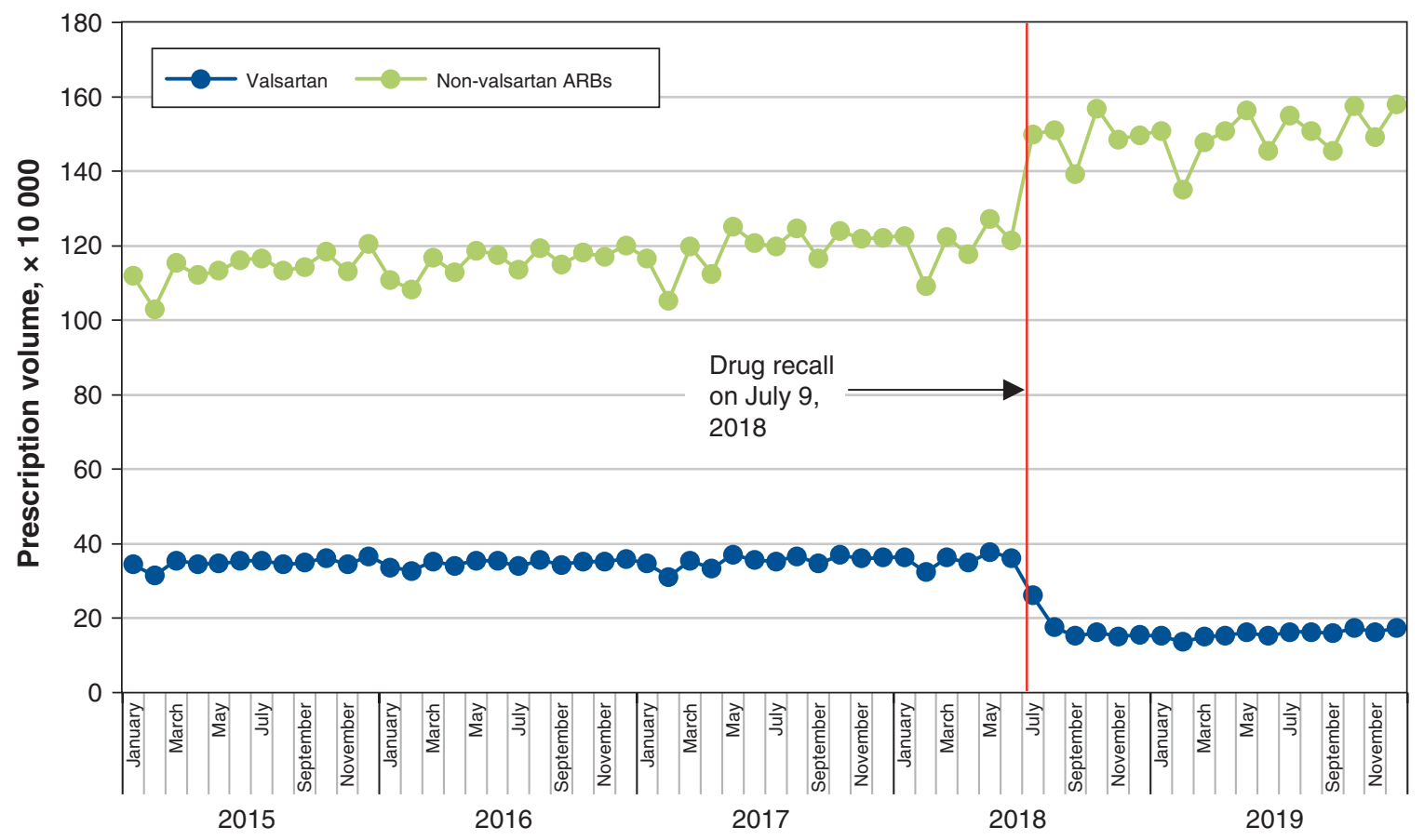

Year and month

Figure 1: Use of valsartan and non-valsartan angiotensin receptor blockers (ARBs) from 2015 to 2019.

Table 1: Use of antihypertensive drugs before and after the valsartan recall on July 9, 2018

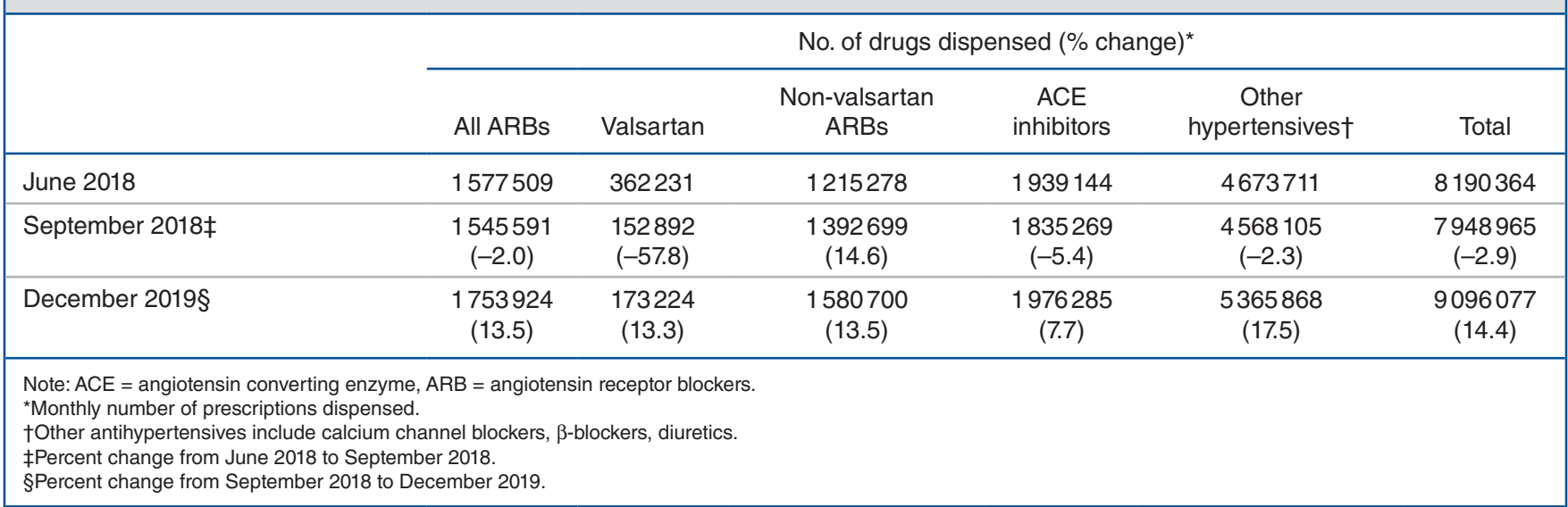

\section{Non-valsartan angiotensin receptor blockers}

Analysis of non-valsartan ARBs dispensed found an increase of $14.6 \%$, from 1215278 prescriptions dispensed in June 2018 to 1392699 in September 2018 (difference = 177421 prescriptions) (Figure 1, Table 1). After this time, non-valsartan ARB dispensing further increased by $13.5 \%$, reaching 1580700 prescriptions in December 2019. The change in non-valsartan ARB prescriptions associated with the valsartan recall in July 2018 was statistically significant $(p<0.0001)$.

\section{Other antihypertensive drug classes}

In the ACE inhibitor and other antihypertensive drug classes, there were 1939144 and 4673711 prescriptions dispensed in June 2018, respectively (Figure 2, Table 1). After the valsartan recall in July 2018, the use of ACE inhibitors decreased 5.4\% (from 1939144 prescriptions in June 2018 to 1835269 in September 2018). However, this reduction was not sustained, with dispensing rising again to 1976285 prescriptions by December 2019. The change in ACE inhibitor prescriptions associated 


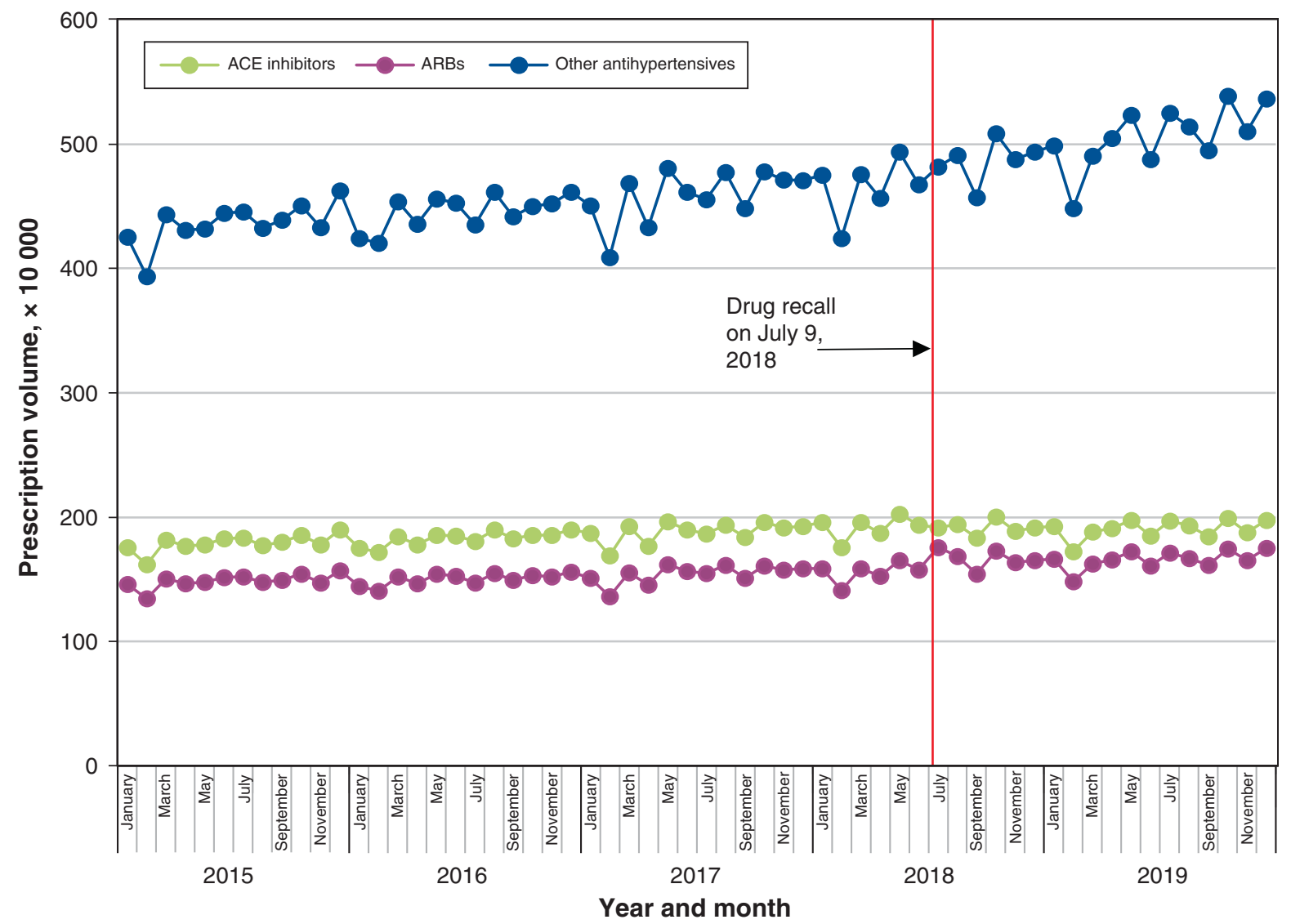

Figure 2: Use of angiotensin receptor blockers (ARBs), angiotensin-converting-enzyme (ACE) inhibitors and other antihypertensives (calcium channel blockers, $\beta$-blockers, diuretics) from 2015 to 2019.

with the valsartan recall in July 2018 was statistically significant $(p<0.0001)$. Use of other antihypertensive drug classes did not change significantly in response to the valsartan recall $(p=$ $0.07)$.

\section{Interpretation}

In this time-series analysis of national trends in antihypertensive prescriptions, we observed an immediate reduction of 209339 valsartan prescriptions within 3 months of the drug recall, affecting an estimated 160000 patients based on a monthly average of 38 to 40 days of supply dispensed per prescription. The shortage led to a small reduction in overall ARB dispensing, driven by a large reduction in valsartan dispensing and shifts toward use of other ARBs. The valsartan shortage illustrates the ripple effect that a drug shortage can have on similar drugs, leading to challenges in maintaining continuity of therapy for patients; this, in turn, may have a negative impact on clinical outcomes and increase costs from use of the health care system. ${ }^{8,23}$

An important finding of our work is the potential clinical ramifications of the recall. Because of the recall, many patients on valsartan were forced to switch to another agent, which is of concern. Altering pharmacotherapy can have serious negative clinical consequences, including adverse drug events, medication errors, increased hospitalizations and death. ${ }^{7,8}$

Further, we found that some valsartan prescriptions did not appear to be replaced with any alternative antihypertensive drug. This is similar to a recent user-level study in Ontario, Canada, that found 1 in 10 patients did not receive replacement for valsartan after its recall. ${ }^{7}$ Although the reasons for stopping medication in our study are not known, potential reasons could include patient fears about the negative consequences of ingesting a recalled medication containing a carcinogen, ${ }^{7}$ or barriers to accessing a health care provider before running out of medication.

Even with a successful change of drug, however, alterations in prescribing may still be accompanied by additional laboratory testing (e.g., serum potassium, renal function), physician visits and communications to ensure that efficacy and safety are retained..$^{15}$ These extra demands may lead to patients experiencing increased stress, frustration and additional travel time to manage alternative therapy. ${ }^{8,15}$ All these factors may be consequential to patients' overall health and 
clinical outcomes. Additional studies are needed to gain a better understanding of how drug shortages affect health care use and their clinical impact on serious adverse outcomes.

The shortage also had broader impacts to the dispensing of the antihypertensives drug class as a whole. In comparison to typical drug shortages that last an average of 4 to 5 months, ${ }^{6}$ the valsartan recall was expanded twice and supply remained reduced for longer than 6 months before it began to recover. Although certain formulations of valsartan were not subject to recall, ${ }^{11,24}$ the low supply of valsartan could not meet increased demands given very limited product availability at that time, causing shifts to other ARBs. We observed a clinical preference of replacing valsartan with another ARB over ACE inhibitors, possibly because ARBs are commonly used in patients who cannot tolerate ACE inhibitors. ${ }^{25,26}$ Decreased use of ACE inhibitors after the recall was most likely related to multiple shortages in the ACE inhibitor drug class between July and September 2018, rather than representing any change in usage patterns. We did not observe any major changes in the other antihypertensive drug groups.

We found that around $50 \%$ of valsartan prescriptions remained substituted at the end of the study period, meaning that patients who moved to another medication likely continued long term. This increased use of other drugs created pressure on the supply chain in other areas, ${ }^{2,15,27}$ with subsequent shortages of several other ARBs during the study time frame caused by increased demand. ${ }^{13}$ The system-wide effects of shortages reflect the limitations of current drug selection and procurement policies and infrastructure, suggesting a strong need for improvement in this area.

Because of the challenges of adapting clinical decisionmaking during a drug shortage, policy-makers should consider some approaches in the future to address this issue. Leveraging the expanded scope of practice of nonphysician health care providers, such as pharmacists and nurse practitioners, to modify therapies for drugs with shortages may improve patient access and allow for more timely responses to patient needs by the health care system. ${ }^{28,29}$

It is also important that regulatory agencies provide timely, reliable and accurate guidance to clinicians and patients affected by drug recalls and shortages, to promote the dissemination of reliable and complete information and to minimize misunderstanding by patients from media reports on the topic. Several studies have raised concerns about the difficulty faced by clinicians in finding suitable alternatives for drugs in short supply. ${ }^{15,30,31}$ In the case of valsartan, other ARBs with structural similarities (i.e., a tetrazole ring) may also have been implicated in carcinogenic contamination, making the navigation of product selection unclear. ${ }^{14,31}$ Therefore, the integration of clinical guidance to assist clinicians with appropriate selection of therapeutic alternatives for drugs in short supply could be beneficial.

Other potential strategies include leveraging provincial or national formularies to develop policies that account for drug shortages as a factor influencing drug selection and procurement. For example, pareto-improving contracts (i.e., contracts that account for the quality of the drug supply chain by pairing drug purchase contracts with failure-to-supply clauses) may lessen the impact of drug shortages; ${ }^{32}$ metrics to incentivize and reward may also financially motivate manufacturers to improve the reliability of their supply. ${ }^{2}$ As the root causes of the valsartan supply issue link back to source ingredients from a pharmaceutical plant in China, more information is needed on the production of active pharmaceutical ingredients, finished dosage forms and policy strategies for multiple sourcing of drugs (i.e., globalization of ingredient manufacturing to include Canada, the United States and Europe), as well as a more coordinated global response to avert future shortages of a similar nature. Although the approach to mitigating and managing shortages will most certainly require a combination of strategies and involvement of broad stakeholders from payers to clinical groups, policy-makers must develop rational solutions to ensure an uninterrupted drug supply.

\section{Limitations}

We could not access patient-specific data, which prevented us from incorporating clinical information (e.g., indication) and sociodemographic characteristics (e.g., age, sex) when analyzing trends in drug use. Without knowing patient diagnoses, we could not investigate whether alternative medications were clinically appropriate substitutions or if reasons for stopping valsartan were appropriate (e.g., polypharmacy, lack of indication), were from adverse effects or were unrelated to the recall (e.g., noncompliance). The data represent prescriptions filled and dispensing volumes, but we could not confirm that the units were consumed. Additionally, the number of affected patients is an estimation based on changes in prescription numbers as we did not have patient-specific data to determine the number of individuals.

\section{Conclusion}

The increasing number of drug shortages, and consequences thereof, remains an unresolved global issue. Our study highlights the potential and substantial impacts of a major drug shortage. The valsartan shortage affected an estimated 160000 patients in Canada, causing rapid shifts in drug use, which in turn likely caused pressure on patients, clinicians and the health care system to find alternative therapies. These findings emphasize the need to investigate the clinical consequences of drug shortages in the population, and to develop improved approaches to mitigate the impact of major shortages.

\section{References}

1. Donelle J, Duffin J, Pipitone J, et al. Assessing Canada's drug shortage problem. Commentary 515. Toronto: C.D. Howe Institute; 2018. doi: 10.2139/ ssrn.3192558.

2. Reed BN, Fox ER, Konig M, et al. The impact of drug shortages on patients with cardiovascular disease: causes, consequences, and a call to action. Am Heart 7 2016;175:130-41.

3. Drug shortages in Canada. Ottawa: Health Canada; modified 2017 Apr. 21. Available: https://www.canada.ca/en/health-canada/services/drugs-health-products/ drug-products/drug-shortages.html (accessed 2021 June 18).

4. Drug shortages: public health threat continues, despite efforts to help ensure product availability Washington (DC): United States Government Accountability Office; 2014. Available: https://www.gao.gov/assets/670/660785.pdf (accessed 2021 June 27).

5. Dill S, Ahn J. Drug shortages in developed countries: reasons, therapeutic consequences, and handling. Eur 7 Clin Pharmacol 2014;70:1405-12.

6. Rinaldi F, de Denus S, Nguyen A, et al. Drug shortages: patients and health care providers are all drawing the short straw. Can f Cardiol 2017;33:283-6. 
7. Jackevicius CA, Krumholz HM, Chong A, et al. Population impact of generic valsartan recall. Circulation 2020;141:411-3.

8. Phuong JM, Penm J, Chaar B, et al. The impacts of medication shortages on patient outcomes: a scoping review. PLoS One 2019;14:e0215837.

9. Videau M, Lebel M, Bussieres J. Drug shortages in Canada: data for 20162017 and perspectives on the problem. Ann Pharm Fr 2019;77:205-11.

10. Several drugs containing valsartan being recalled due to contamination with a potential carcinogen. Ottawa: Health Canada; modified July 10. Available: https:// www.healthycanadians.gc.ca/recall-alert-rappel-avis/hc-sc/2018/67202a-eng.php (accessed 2021 June 18).

11. Expansion of recall of valsartan drugs to include additional lots, as a precaution. Ottawa: Health Canada; modified 2018 Dec. 18. Available: https:// healthycanadians.gc.ca/recall-alert-rappel-avis/hc-sc/2018/67552a-eng.php? $\mathrm{ga}=2.186111849 .1130786600 .1536540324-1989020253.1520525748$ (accessed 2021 June 18).

12. Farrukh MJ, Tariq MH, Malik O, et al. Valsartan recall: global regulatory overview and future challenges. Ther Adv Drug Saf 2019;10:2042098618823458.

13. Drug shortages homepage. Ottawa: Health Canada. Available: https://www. drugshortagescanada.ca/ (accessed 2021 June 18).

14. Gillette $M$, Taylor A, Butulija D, et al. Reflections of the angiotensin receptor blocker recall by the FDA and repercussions on healthcare. Cardiovasc Drugs Ther 2020;34:579-84.

15. Byrd JB, Chertow GM, Bhalla V. Hypertension hot potato: anatomy of the angiotensin-receptor blocker recalls. NEngl 7 Med 2019;380:1589-91.

16. Schwartz KL, Chen C, Langford BJ, et al. Validating a popular outpatient antibiotic database to reliably identify high prescribing physicians for patients 65 years of age and older. PLoS One 2019;14:e0223097.

17. Tran Q, Warren JL, Barrett MJ, et al. An evaluation of the utility of big data to supplement cancer treatment information: linkage between IQVIA pharmacy database and the Surveillance, Epidemiology, and End Results Program. 7 Natl Cancer Inst Monogr 2020;2020:72-81.

18. Black CD, McCarthy L, Gomes T, et al. Interprovincial variation of psychotropic prescriptions dispensed to older Canadian adults. Can Geriatr 72018 ; 21:269-73.

19. Xu Y, Gomes T, Mamdani MM, et al. Analysis of trends in insulin utilization and spending across Canada from 2010 to 2015. Can 7 Diabetes 2019;43:179-85.e1.

20. Helfenstein $U$. The use of transfer function models, intervention analysis and related time series methods in epidemiology. Int 7 Epidemiol 1991;20:808-15.

21. Dickey D, Fuller W. Distribution of the estimators for autoregressive time series with a unit root. 7 Am Stat Assoc 1979;74:427-31.

22. Ljung G, Box G. On a measure of lack of fit in time series models. Biometrika 1978;65:297-303.

23. Shaban H, Maurer C, Willborn RJ. Impact of drug shortages on patient safety and pharmacy operation costs. Fed Pract 2018;35:24-31.

24. Leclerc J. Recall of N-nitrosodimethylamine-contaminated pseudogeneric valsartan: Best generics finally no better than others? Can 7 Cardiol 2018;34:1370.e13.

25. Caldeira D, David C, Sampaio C. Tolerability of angiotensin-receptor blockers in patients with intolerance to angiotensin-converting enzyme inhibitors: a systematic review and meta-analysis. Am 7 Cardiovasc Drugs 2012;12:263-77.

26. Yilmaz I. Angiotensin-converting enzyme inhibitors induce cough. Turk Thorac 7 2019;20:36-42.

27. Elbeddini A, Hooda N, Yang L. Role of Canadian pharmacists in managing drug shortage concerns amid the COVID-19 pandemic. Can Pharm 7 (Ott) 2020;153:198-203.

28. Jebara T, Cunningham S, MacLure K, et al. Stakeholders' views and experiences of pharmacist prescribing: a systematic review. Br 7 Clin Pharmacol 2018;84:1883-905.
29. Courtenay M. Nurse prescribing, policy, practice and evidence base. $\operatorname{Br} \mathcal{F}$ Community Nurs 2008;13:563-6.

30. Special issue: Drug shortages - National survey reveals high level of frustration, low level of safety. Horsham (PA): Institute for Safe Medication Practices (ISMP); 2010. https://www.ismp.org/resources/special-issue-drug-shortages -national-survey-reveals-high-level-frustration-low-level (accessed 2021 June 18).

31. Gunasekaran PM, Chertow GM, Bhalla V, et al. Current status of angiotensin receptor blocker recalls. Hypertension 2019;74:1275-8.

32. Jia J, Zhao H. Mitigating the US drug shortages through pareto-improving contracts. Prod Oper Manag 2017;26:1463-80.

Affiliations: Leslie Dan Faculty of Pharmacy (Fenna, Hassan, Gomes, Tadrous), University of Toronto, Toronto, Ont.; Pharmacy Services (Fenna), Alberta Health Services, Edmonton, Alta.; Institute of Health System Solutions and Virtual Care (Chu, Tadrous), Women's College Hospital; Li Ka Shing Knowledge Institute (Gomes), Unity Health Toronto; Institute of Health Policy, Management and Evaluation (Gomes), University of Toronto, Toronto, Ont.

Contributors: All of the authors contributed to the conception and design of the work. Jennifer Fenna and Mina Tadrous acquired and analyzed the data. All of the authors contributed to data interpretation. All of the authors drafted the manuscript, revised it critically for important intellectual content, gave final approval of the version to be published and agreed to be accountable for all aspects of the work.

Funding: This study was funded by a grant from the Ontario Ministry of Health's Health System Research Fund.

Content licence: This is an Open Access article distributed in accordance with the terms of the Creative Commons Attribution (CC BY-NC-ND 4.0) licence, which permits use, distribution and reproduction in any medium, provided that the original publication is properly cited, the use is noncommercial (i.e., research or educational use), and no modifications or adaptations are made. See: https://creativecommons.org/licenses/ by-nc-nd/4.0/

Data sharing: This study was an analysis of a claims database using data obtained under licence from IQVIA Canada Inc. The raw data cannot be publicly shared as it was obtained from a third party and as per signed agreement. Requests for data can be sent to IQVIA Solutions Canada Inc. and may carry a cost.

Disclaimer: The statements, findings, conclusions, views and opinions contained and expressed in the report are based in part on data obtained under licence from IQVIA, Canada Inc. concerning the following information services: GPM, data period Jan. 1, 2015, to Dec. 31, 2019. All rights reserved. The statements, findings, conclusions, views and opinions expressed herein are not necessarily those of IQVIA Canada Inc. or any of its affiliated or subsidiary entities.

Supplemental information: For reviewer comments and the original submission of this manuscript, please see www.cmajopen.ca/content/9/4/ E1128/suppl/DC1. 